\title{
Supervisory Control System for Adaptive Phase and Work Cycle Management of Sequencing Wastewater Treatment Plant
}

\author{
P. HIRSCH, R. PIOTROWSKI, K. DUZINKIEWICZ, M. GROCHOWSKI \\ Gdansk University of Technology, Faculty of Electrical and Control Engineering, \\ Narutowicza 11/12, 80-233 Gdansk, Poland, \\ piotr.hirsch@pg.edu.pl, robert.piotrowski@pg.edu.pl, \\ kazimierz.duzinkiewicz@pg.edu.pl,michal.grochowski@pg.edu.pl
}

\begin{abstract}
The paper presents the design of the integrated control system applied to Sequencing Batch Reactor (SBR) in a biological Wastewater Treatment Plant (WWTP) in Swarzewo, which operates under activated sludge technology. Based on the real data records, ASM2d biological processes model and aeration system model, hierarchical control system for dissolved oxygen tracking and cycle management is designed. Internal Model Controller (IMC) was applied to control the air flow at the lower control level. Higher level dissolved oxygen controller is based on Direct Model Reference Adaptive Control (DMRAC) method. The supervisory system performs management of reactor work cycle, determines the phase length, controls sludge age, calculates setpoint of dissolved oxygen and adapts parameters of the lower control layer. Proposed control system allowed to: increase the efficiency, improve the quality of outflow and reduce the cost of aeration and chemical treatment plant, in relation to existing solutions in case study plant.
\end{abstract}

Keywords: activated sludge, aeration, control, wastewater treatment, dynamic simulation, sequencing batch reactor.

\section{Introduction}

\subsection{General information}

In industrial practice, two different types of activated sludge Wastewater Treatment Plant (WWTP) are used: Sequencing Batch Reactor (SBR) and WWTP with a continuous flow throughout the plant. In SBR, all biochemical processes occurs in one tank, in the predefined sequence. In the second type of plant, several tanks are connected by recirculation flows (internal and external). In this paper the SBR type of the WWTP is considered.

The SBR technology is widely used under small wastewater inflows conditions and may be designed using a single or multiple tanks in parallel. A typical work cycle involves five operational phases: filling, biochemical reactions (aerobic, anoxic, anaerobic), sedimentation, decantation and idle state.

A variety of different control strategies may be set up by selecting of number and sequence of SBR operational phases, in order to adjust SBR to specific working conditions of each WWTP. Because of this SBR gains operational flexibility, that allows this kind of WWTP to benefit from control system modernization. It has been shown that SBR pollutant removal efficiency can be greatly improved by application of step-feed control strategy [16]. A variant of this strategy is implemented in
Swarzewo WWTP, Northern Poland, which is the case study considered in this paper. During the biochemical reaction phases, pollutions in the form of organic matter and nutrients (mainly phosphorus and nitrogen compounds) are degraded during the mineralization, nitrification, denitrification and enhanced biological phosphorus removal. Efficiency of those processes relay strongly on oxygen concentration, therefore high quality of DO control is crucial for fulfilment of discharge limits. The DO control is also important for WWTP energy efficiency, as the aeration system is responsible for $50-75 \%$ of plant power usage [1].

The supervisory control in continuous flow and SBR WWTPs is of a different nature. The main objective of the continuous flow WWTP supervisory controller is to determine the oxygen setpoint and recirculation flows, that will be sufficient to efficiently treat the wastewater. In the case of the SBR, objective of the supervisory controller is to manage the work cycle, namely the sequence and length of operational phases. Therefore SBR supervisory controller may be called work cycle controller.

\subsection{Survey of related works}

Broad range of control technologies have been researched and applied to DO control, e.g. adaptive controller $[4,9,13]$, predictive 
controller $[5,7,11,12,18]$, multivariable PID controller [19], fuzzy controller [3,14], fuzzy predictive controller $[15,20]$. Extensive review of DO control can be found in [1].

In [4] Direct Model Reference Adaptive Control (DMRAC) technology for DO tracking in Swarzewo case study was presented. Furthermore, analysis of the parameters of the adaptive controller for control quality was examined [13]. The authors used calibrated SBR Activated Sludge Model No. 2d (ASM2d) [8] coupled with validated aeration system model [14]. Simulations proved that the proposed solution provides satisfactory control quality in the case of most basic work cycles based on single filling pattern [4]. However, application of more complex cycle control strategies showed that discussed control system lacks adaptability. Classic DMRAC is ineffective when applied to SBR operating with step-feed strategy in occurrence of influent fluctuations. Hence, additional stepwise adaptation of DMRAC parameters (learning rates, anti-windup filter gains and starting points) was applied and examined in [9].

The most basic applications of SBR work cycle controllers are based on a fixed duration and composition of the work cycle and subsequent phases. Numerous studies were conducted on the optimal selection of these scenarios. In [2] the effect of total cycle time, phases duration and phase sequence on nutrient removal performance were examined. Systematic approach to examine and to determine the optimal operation strategy for nutrient removal was developed [16]. On-line method of phase duration optimization was presented in [10]. Discussed algorithm was based on measurements of oxidation reduction potential, dissolved oxygen and $\mathrm{pH}$. Upon these measurements, optimal end points of aerobic and anoxic phases were determined. In 2005 aerobic phase duration optimization was presented [17]. Authors examined possibility of using oxygen uptake rate measurements alongside fuzzy controller to determine nitrification termination.

\subsection{Problem statement and main contribution}

In order to take full advantage of the SBR capabilities, it is necessary to apply complex work cycle composition with the intermittent filling method. The overview of related works shows, that attempts were made to determine an optimal work cycle composition, but with an assumption of fixed cycle duration. Similarly, methods of optimal phase design were researched with an assumption of fixed work cycle composition. In this paper an attempt to combine the advantages of these two approaches is made. Supervisory controller is proposed for the on-line phase duration optimization with adaptive work cycle structure arranging. Moreover, DO controller that takes into account limitations of aeration system is applied in the control structure.

In most of previously described research works the dynamics and limitations of aeration system were omitted. However, the impact of aeration system on performance and control strategies of WWTP can be significant. This applies for the SBR especially, as the aeration system has to work in wide pressure range due to step-feed strategy used in this kind of plants. In this paper nonlinear dynamics and limitations of aeration system were coupled with biological processes in the SBR.

In summary, the aim of this paper is to design the supervisory control system for adaptive phase and work cycle management of SBR, in wide operating range. This paper further develops the research works presented in $[4,9,13]$.

\section{Plant Description and Modelling}

WWTP at Swarzewo is a typical SBR plant. The average WWTP wastewater influent is about $6000 \mathrm{~m}^{3} /$ day. Wastewater treatment involves mechanical, biological and chemical processes. Separation of sewage solids takes place in the mechanical part (grid, screen, grit chamber, sand separator). The chemical part is used to remove phosphorus from wastewater using coagulants. Nutrient removal is performed by four SBRs. SBR capacities are as follows: SBR 1, 2, 3 are of $5100 \mathrm{~m}^{3}$, SBR 4 is of $4948 \mathrm{~m}^{3}$. They operate independently and in parallel. Air supply for the reactors is fed by two separate aeration systems. SBRs 1, 2 and 3 are supplied by the first, SBR 4 by the second. Detailed description of WWTP at Swarzewo can be found in [4].

The ASM2d model, presented by International Water Association task group [8], was used to simulate the plant. The model was calibrated 
based on real data sets from WWTP at Swarzewo. Additionally, the values of: chemical oxygen demand (COD), total nitrogen $\left(\mathrm{N}_{\text {tot }}\right)$ and total phosphorous $\left(\mathrm{P}_{\text {tot }}\right)$ were determined basing on [8]. The aeration system is a complicated, nonlinear, dynamic system itself. The reactor considered in this study (reactor 4) is supplied by the aeration system composed of a blower station, collecting pipe, two diffuser systems and collector-diffuser pipes. General methodology for aeration system modelling was presented in [11]. This approach was applied for different aeration systems e.g. [4,5,9,12-14]. Identification and validation tests for the case study aeration system were described in [14], as well as, modifications and final version of the model.

\section{Control Strategies}

\subsection{Control structure}

The DO and aeration system dynamics are nonlinear and hence, good control performance over all operating conditions cannot be expected to be achieved with a conventional linear controller. In addition, the dynamic of biological processes have different time scales. The hierarchical control structure as presented in Figure 1 is proposed. Supervisory controller at the higher level is responsible for setting a DO reference trajectory, counting the current fill phase, managing the work cycle (by phase control) and controlling the sludge age. At the lower level cascade controller is proposed, with air flow being controlled in the inner loop and DO concentration in the outer one. Cascade structure fits considered case, since DO has nonlinear dynamic with two distinguishable time scales (seconds for air flow, several minutes for DO concentration) and distinct intermediate variable (air flow).
On behalf of having precise and verified model of Swarzewo aeration system, Internal Model Control (IMC) technique was proposed for inner loop of the cascade controller (air flow controller - see Figure 1). The concept of IMC was first formalized by [6] and can be applied to nonlinear and multivariable systems. The IMC operation principle refers to the transformation of the reference signal into the control signal by the inverted model of the controlled plant. The transformation could be performed in an open loop structure, but due to the aeration system certain time-variance, a closed loop approach was applied in order to prevent control quality deterioration caused by possible model inaccuracy. Due to much faster response of the inner loop (air flow/aeration system) than the outer loop (DO concentration/SBR), static model of aeration system for IMC was used. This has allowed significant simplification of the air flow controller without apparent impact on DO control quality. In such case inverted model of aeration system was obtained by the means of inverted static characteristic of the original aeration system. Considered aeration system comprises two blowers, therefore, prior to static characteristic determination, blowers toggle mode has to be specified. Independent work of both blowers is considered. During each aeration phase one of the blowers is regarded as the main (hereinafter called $B_{M}$ ) and the other as the auxiliary $\left(\mathrm{B}_{\mathrm{A}}\right) . \mathrm{B}_{\mathrm{A}}$ is launched only to complement the air flow generated by $\mathrm{B}_{\mathrm{M}}$, when it is below the set point. When the $B_{M}$ load falls below $86 \%$ of the maximum performance (calculated with respect to the speed) $B_{A}$ is turned off. $B_{M}$ can also be turned off if the air flow set point is less than $2 / 3$ of the minimum air flow possible to generate at a given pressure. At the same time, a time limit on the blower switching frequency is

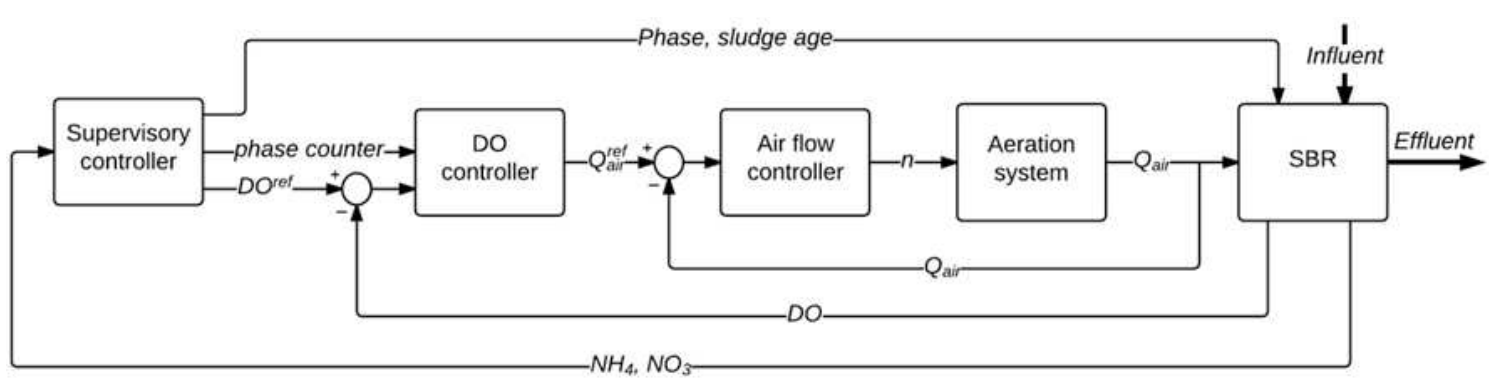

Figure 1. Structure of hierarchical control system where: $Q_{\text {air }}$ denotes air flow $\left[\mathrm{m}^{3} / \mathrm{h}\right], n$ stands for blower speed [rpm], and ref is abbreviation for reference trajectory, $\mathrm{NH}_{4}$ and $\mathrm{NO}_{3}$ are ammonia $\left[\mathrm{g} \mathrm{N} / \mathrm{m}^{3}\right]$ and nitrate $[\mathrm{g}$ $\mathrm{N} / \mathrm{m}^{3}$ ] concentrations in the SBR, respectively. 
considered. After turning off, the blower can be restarted only after 5 minutes. Whenever the $B_{M}$ is being turned off (due to e.g. the end of the aeration phase) the roles of blowers are exchanged. Detailed description of the IMC can be found in [14].

A modified Direct Model Reference Adaptive Controller (DMRAC) was proposed to control the DO at the outer loop of the cascade (Figure $1)$. It is presented in details in $[4,9]$, including stability analysis.

\subsection{Supervisory controller}

\subsubsection{Work cycle}

There is a great number of the possible modifications of the basic SBR cycle described in section 1.1., but regardless of the approach, some elements are fixed. The cycle begins with the filling phase, when a portion of wastewater enters reactor. Afterwards a sequence of biological reactions is carried out, with the possibility of additional filling. Next step is sedimentation followed by discharge of the treated supernatant (decantation) and the waste of excess sludge. Methodology for the designing of the work cycle is proposed basing on the following order of consideration: filling conditions, the last biological reaction in the cycle, the biological reactions sequence, the number of the sequences in the cycle.

Filling phase can be carried out either under aerobic or anoxic conditions. The latter is the most commonly applied one and it will be applied in presented case. There are strong reasons for such an approach - raw sewage provides the carbon needed for the denitrification. In the course of intermittent filling under anoxic conditions, nitrite and nitrate remaining after the last cycle or remaining after the last aerobic phase can be effectively removed without any additional, external sources of carbon.

It is convenient to analyse the sequence of biological reactions from the end that is from the last biological reaction. If it is aerobic, the $\mathrm{NO}_{3}$ concentration in the effluent will be increased, if anoxic, there will be more $\mathrm{NH}_{4}$. Taking into account enhanced biological phosphorus removal and fact that polyphosphate accumulating organisms release phosphates under anaerobic conditions, it was decided that the last reaction phase should be aerobic. Thereby, the risk of re-release of phosphates during sedimentation and decantation phases is lowered and overall phosphates concentration in effluent decreases. Stabilization ponds are the endpoint of the technological chain in Swarzewo WWTP. Anoxic conditions present in ponds are conducive to denitrification, which is an additional argument for choice of the last biological reaction.

After defining the last biological reaction and filling conditions, the entire sequence can be determined. Previously adopted principles are naturally complemented by an anoxic reaction placed after filling and before aeration. Following sequence will be thus obtained: filling $=>$ anoxic reaction $=>$ aerobic reaction. Such sequence composition has numerous advantages resulting from biological nutrient removal conditions. Adding a portion of raw wastewater immediately before each anoxic reaction provides the carbon required for the denitrification and phosphorus removal. Anoxic conditions initiated during the filling phase are transformed into anaerobic conditions at the end of the anoxic reaction, which is also an essential condition for enhanced biological phosphorus removal.

In $[2,16]$ research on the filling strategy of the SBR and the number of biological reactions sequences were conducted. Each time the results have indicated improvement in the nutrient removal efficiency with the introduction of intermittent filling and multiple biological reaction sequences. Depending on the working conditions of given WWTP, it is assumed that the system works best with 4-8 sequences. It should be noted that in each of these works, fixed work cycle duration was assumed. It can be argued that in the case of fixed cycle and phases duration, increasing the number of sequences is essential for improving the quality of outflow. From the analysis of the results presented in [10, 17] it was concluded that in the case of phase duration optimization, the quality of the effluent is dependent rather on the volume of the last filling than on the number of sequences. Simulation studies were applied in order to verify this thesis. The simulation is described in detail in section 4 and the results are shown in the Table 1.

The comparison of the experiments characterized by the same number of sequences and different volumes of the last filling (experiments 3 and 4,5 and 6,7 and 8) shows, 
Table 1. Number of sequences and last filling volume impact on effluent quality.

\begin{tabular}{|c|c|c|c|c|c|}
\hline Run & $\begin{array}{c}\text { Sequences } \\
\text { number }\end{array}$ & $\begin{array}{c}\text { Last filling } \\
\text { volume }\left[\mathbf{m}^{3}\right]\end{array}$ & $\begin{array}{c}\mathbf{C O D} \\
{\left[\mathbf{g ~ \mathbf { O } _ { 2 }} / \mathbf{m}^{\mathbf{3}}\right]}\end{array}$ & $\begin{array}{c}\mathbf{N}_{\text {tot }} \\
{\left[\mathbf{g ~ N} / \mathbf{m}^{3}\right]}\end{array}$ & $\begin{array}{c}\text { Cycle } \\
\text { duration }[\mathbf{h}]\end{array}$ \\
\hline $\mathbf{1}$ & 9 & 167 & -131.5 & 3.13 & 27.77 \\
\hline $\mathbf{2}$ & 6 & 159 & -132.8 & 3.5 & 26.1 \\
\hline $\mathbf{3}$ & 4 & 444 & -143.8 & 7.36 & 22.68 \\
\hline $\mathbf{4}$ & 4 & 64 & -135.5 & 3.77 & 27.98 \\
\hline $\mathbf{5}$ & 3 & 406 & -144.4 & 7.68 & 23.09 \\
\hline $\mathbf{6}$ & 3 & 151 & -142 & 5.38 & 29.14 \\
\hline $\mathbf{7}$ & 2 & 869 & -167.1 & 15.25 & 20.27 \\
\hline $\mathbf{8}$ & 2 & 490 & -154 & 10.04 & 24.7 \\
\hline
\end{tabular}

that $\mathrm{N}_{\text {tot }}$ in the effluent significantly increases with the increasing of the last filling volume. Simultaneously, comparison of the experiments $1,2,3$ and 5 shows that the outflow quality is not notably improved just by increase in the number of sequences.

Correlation between the cycle duration and the volume of the last filling can be found by comparing experiments with the same number of sequences ( 3 and 4,5 and 6,7 and 8). Total cycle duration significantly increases, when the volume of the last filling decreases. This phenomenon is due to the fact that the last filling volume did not provide enough carbon for the effective denitrification, thereby lengthening the denitrification phase. Forcing the appropriate volume of the last filling is crucial in terms of the cycle duration and outflow quality.

In very simplistic terms, it can be assumed that the processes of nitrification and denitrification have inertial character. This means that, in the general case, the duration of the cycle with the phase optimization will increase in the case of more sequences. This situation is represented by the experiments 1,2 and 3 (Table 1).

Taking these results into account, it was decided to adopt the strategy of asymmetric filling with the varying number of the biological reactions sequences. The principle is to control the filling with time and volume limits at the same time. The role of the time limit is to divide the cycle into number of sequences depending on the intensity of wastewater inflow. If the flow rate is smaller, more sequences occur, what will take more time respectively. Whereas large inflows require rapid treatment, and so the cycle will consist of less sequences. The role of the volume limit is to force a particular volume of the last filling, regardless of the intensity of the wastewater inflow. The block diagram illustrating the course of the cycle established for Swarzewo is given in Figure 2, where: $t_{f}$ is the duration of the current filling [min]; $t_{f, \max }$ is a time limit on filling [min]; $t_{s}$ is the duration of sedimentation [min]; $t_{s, \max }$ is a time limit on sedimentation [min]; $t_{w}$ is the duration of excess sludge wasting [min]; $t_{w, \max }$ is a time limit on excess sludge wasting [min]; $V$ is the current volume of the wastewater-sludge mixture in the tank $\left[\mathrm{m}^{3}\right] ; V_{\max }$ is maximum capacity of the tank $\left[\mathrm{m}^{3}\right] ; V_{\min }$ is the minimum volume of the mixture in the tank $\left[\mathrm{m}^{3}\right] ; V_{f}$ is a volume of the last filling $\left[\mathrm{m}^{3}\right] ; \mathrm{NO}_{3}$ is nitrite and nitrate concentrations measure and $N O_{3 \max }$ is a limit on these concentrations $\left[\mathrm{g} \mathrm{NO}_{3}+\mathrm{NO}_{2} / \mathrm{m}^{3}\right] ; \mathrm{NH}_{4}$ is ammonium concentration measure and $\mathrm{NH}_{4 \max }$ is its limit $\left[\mathrm{NH}_{4} \mathrm{~g} / \mathrm{m}^{3}\right] ; T S S_{1}$ is a concentration of the Total Solid Suspension (TSS) in the top layer of the tank and $T S S_{\text {Imin }}$ is its limit $\left[\mathrm{g} / \mathrm{m}^{3}\right]$; $T S S_{e}$ is a concentration of the TSS in the effluent and $T S S_{\text {emax }}$ is its limit $\left[\mathrm{g} / \mathrm{m}^{3}\right] ; d T S S_{2}$ derivative of the TSS concentration in the middle layer of the reactor $\left[\mathrm{g} / \mathrm{m}^{3}\right]$.

\subsubsection{Phase management}

The SBR phase management can be based either on the fixed scenario or on the measurements. The former approach requires manual corrections on the duration of individual phases in order to adjust work cycle to varying influent and environmental conditions. Phase management based on the measurements allows to terminate each phase when corresponding biological reactions are completed. Therefore, it is called the phase optimization or the optimal phase design. Phase optimization requires appropriate measuring equipment and expert knowledge for the selection of the limit values. Due to the large number of degrees of freedom and mutual 
interactions, selection process is organized in accordance to the work cycle block diagram presented in Figure 2.

In Swarzewo WWTP the wastewater inflow rate is not controlled. Hence, filling phase can be managed only by time and volume limits. In the presence of inflow rate variations, fixed filling time leads to variable number of biological sequences and varying duration of the cycle in consequence. Thereby selection of the $t_{f}, V_{\min }$ and $V_{f}$ parameters directly affects performance of the SBR. Maximum aeration system output have to be considered when specifying these parameters. If the ratio of newly introduced wastewater to mixture already present in the tank is too large, the oxygen uptake rate will exceed the capabilities of the aeration system, thus, leading to suboptimal conditions for nitrification and prolonging of the aeration phase. The volume of the last filling has to be specified in accordance to filling time, as it provides carbon for denitrification of the $\mathrm{NO}_{3}$ produced during previous sequence. The $V_{\min }$, which determines the volume of the wastewater treated during the cycle, is limited by the sludge volume and oxygen uptake rate.

The aerobic reaction finishes when $\mathrm{NH}_{4}$ concentration is close to zero. Correspondingly, anoxic/anaerobic reaction ends with the lack of the $\mathrm{NO}_{3}$. The instance of the depletion of corresponding nitrogen compound can be directly specified at Swarzewo WWTP, as there are probes for ammonium and nitrate concentration measurements installed. Given that, it is enough to specify $\mathrm{NH}_{4}$ and $\mathrm{NO}_{3}$ limit values close to zero.

Sedimentation phase is managed on the basis of the total suspended solids measurements in the top and the middle layer of the reactor. It is important to not prolong the sedimentation time, as it may lead to return discharge of phosphates into treated wastewater. The $t_{s, \max }$ parameter is selected to enable the sludge to thicken at the bottom of the tank before excess sludge wasting. The decantation phase is terminated when volume of the mixture reaches its minimum level, or there are solids detected in the effluent. The values of discussed parameters are shown in Table 2.

\subsubsection{Sludge retention time}

Sludge Retention Time (SRT) is the average period of time the activated sludge solids has remained in the system. It is crucial parameter for activated sludge design as it affects several factors such as: type of the microorganisms in the biomass, the sludge mass and sludge load,

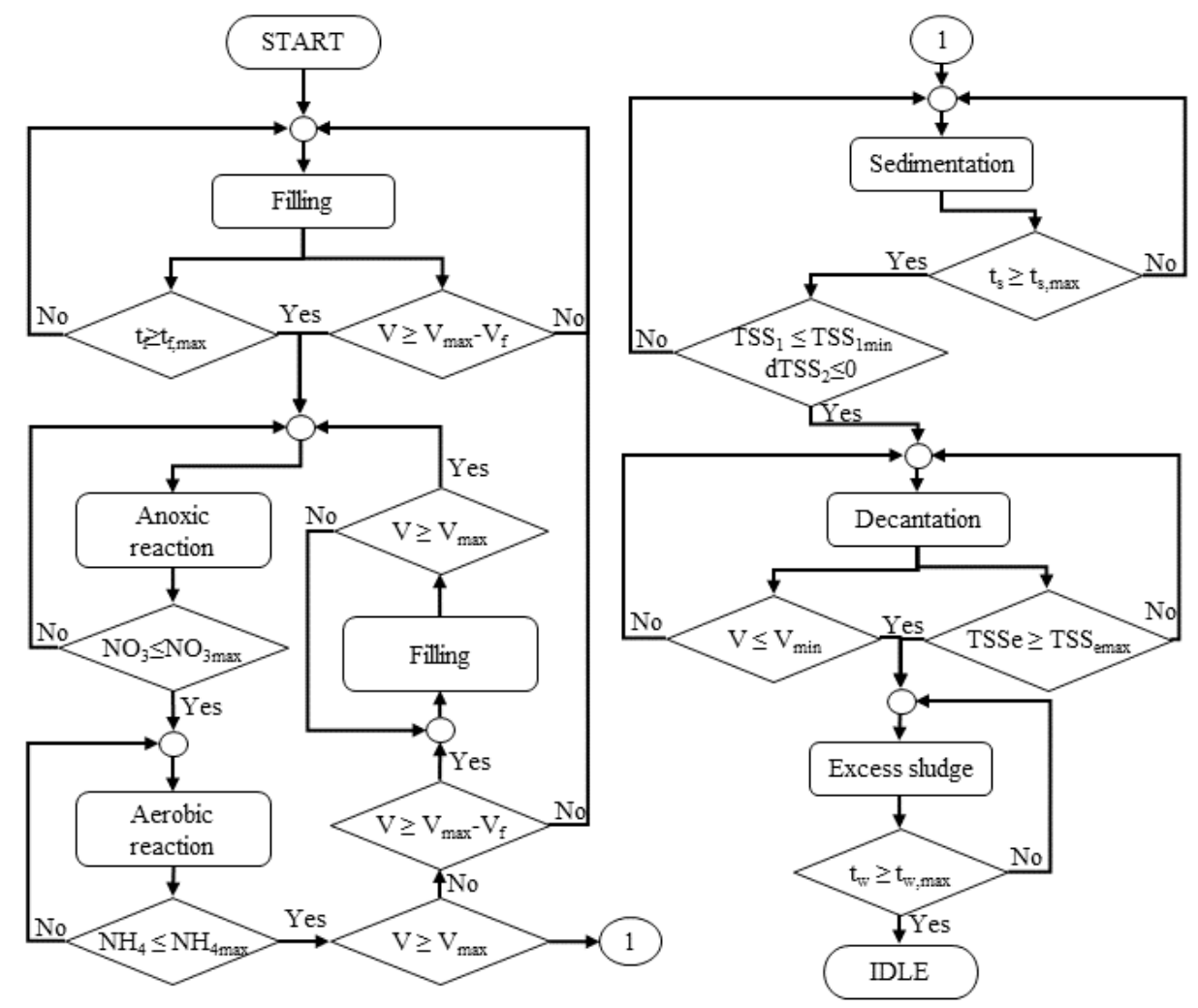

Figure 2. Block diagram of the work cycle. 
Table 2. Phase management limits for Swarzewo WWTP.

\begin{tabular}{|c|c|c|c|c|c|c|c|c|c|c|c|c|c|}
\hline Parameter & $\mathbf{t}_{f_{\max }}$ & $\mathbf{V}_{\mathrm{f}}$ & $\mathrm{NO}_{3 \max }$ & $\mathbf{N H}_{4 \max }$ & $\mathbf{t}_{\mathrm{s} \max }$ & $\mathbf{T S S}_{1 \min }$ & $\mathbf{T S S}_{\text {emax }}$ & $\mathbf{V}_{\min }$ & $\mathbf{V}_{\max }$ & $\mathbf{Q}_{\mathrm{e}}$ & $\mathbf{q}_{\mathrm{w}}$ & $\mathbf{N}_{\mathbf{c}}$ & SRT \\
\hline Value & $\begin{array}{l}120 \\
\min \end{array}$ & $\begin{array}{c}250 \\
\mathrm{~m}^{3}\end{array}$ & $\begin{array}{l}0.2 \mathrm{~g} \\
\mathrm{~N} / \mathrm{m}^{3}\end{array}$ & $\begin{array}{l}0.5 \mathrm{~g} \\
\mathrm{~N} / \mathrm{m}^{3}\end{array}$ & $75 \mathrm{~min}$ & $\begin{array}{c}0.5 \\
\mathrm{~g} / \mathrm{m}^{3}\end{array}$ & $\begin{array}{c}0.5 \\
\mathrm{~g} / \mathrm{m}^{3}\end{array}$ & $\begin{array}{c}4000 \\
\mathrm{~m}^{3}\end{array}$ & $\begin{array}{c}4948 \\
\mathrm{~m}^{3}\end{array}$ & $\begin{array}{c}948 \\
\mathrm{~m}^{3} / \text { cycle }\end{array}$ & $\begin{array}{c}504 \\
\mathrm{~m}^{3} / \mathrm{h}\end{array}$ & $\begin{array}{c}2 \\
\text { cycle/d }\end{array}$ & $32 \mathrm{~d}$ \\
\hline
\end{tabular}

respiration rate or sludge settling. Knowing the TSS in different sections of the reactor, and specific SRT to maintain, $Q_{w}$ is given by (1):

$$
Q_{W}=\frac{V_{\max } \cdot T S S}{S R T \cdot T S S_{3} \cdot N_{c}}-\frac{Q_{e} \cdot T S S_{e}}{T S S_{3}}
$$

where: $S R T$ - sludge (solids) retention time [d], TSS - Total suspended solids (TSS) in the tank $\left[\mathrm{g} / \mathrm{m}^{3}\right], Q_{w}$ - wasted excess sludge $\left[\mathrm{m}^{3} /\right.$ cycle], $T S S_{3}$ - Total Solid Suspension (TSS) at the bottom of the tank $\left[\mathrm{g} / \mathrm{m}^{3}\right], N_{c}$ - the number of the cycles per day, $Q_{e}$ - outflow of the treated wastewater $\left[\mathrm{m}^{3} /\right.$ cycle $]$.

The time of excess sludge wasting $\left(t_{w}\right)$ is determined at the beginning of the decantation phase. It is result of $Q_{w}$ division by the excess sludge pump capacity $q_{w}$. Some of the parameters values result from devices specification, for example $Q_{e}$ and $q_{w}$. The $N_{c}$ value is due to Volumetric Exchange Ratio $V E R=\frac{V_{\max }-V_{\min }}{V_{\max }}$ and the overall plant efficiency. The SRT value was determined on the basis of the simulation studies. Numerical values are shown in the Table 2 .

\subsubsection{Phase counter and $\mathrm{DO}^{\text {ref }}$}

The DO controller utilized in the proposed control structure (Figure 1) has its parameters adapted according to the current aerobic phase. This mechanism allows to adjust the DMRAC properties in order to improve its performance when dealing with step-feed strategy. The successive aerobic phases in a cycle are counted by the supervisory controller, the counter is then forwarded to the DO controller.

During the analysis of possible $\mathrm{DO}^{\text {ref }}$ trajectory scenarios, the aeration system operating range has to be taken into account. As the respiration rate decreases with the duration of the aeration phase, it eventually becomes lower then minimal aeration system output. Therefore, maintaining low DO setpoints would result in frequent blower switching and hence DO oscillations. Therefore, only $1 \mathrm{~g} \mathrm{O}_{2} / \mathrm{m}^{3}$ and higher DO setpoints were considered. The optimal DO level for nitrification is in general $2 \mathrm{~g} \mathrm{O}_{2} / \mathrm{m}^{3}$, however some variations of this value may be advisable depending on the sludge temperature, wastewater composition and biomass condition. Management of the DO concentration is also important in terms of energy consumption and overall WWTP efficiency, understood as the amount of $\mathrm{m}^{3}$ of wastewater treated per day. In general case, increased value of DO concentration will result in decreased aerobic phase duration and increased energy consumption. Electric power consumption to cycle duration for several constant $\mathrm{DO}^{\text {ref }}$ were obtained via simulation. The results are presented in Figure 3.

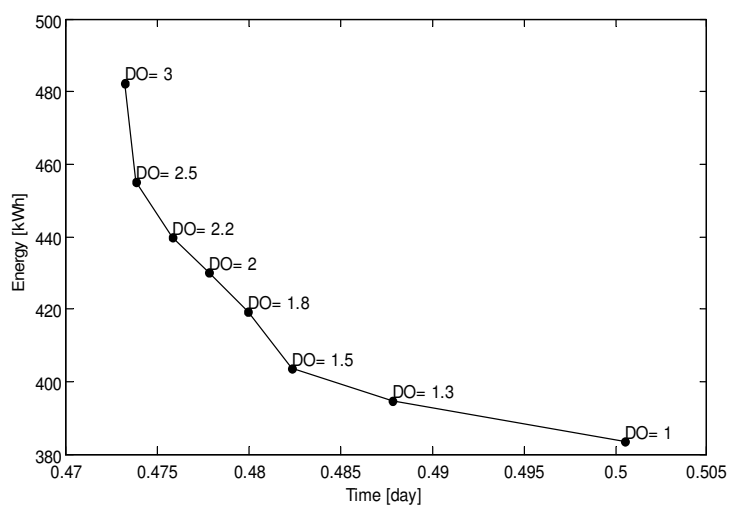

Figure 3. DO concentration influence on electric power consumption and cycle length.

It can be observed that the ratio between electric power usage and the cycle length is approximately linear in the $1.5-2.5 \mathrm{~g} \mathrm{O}_{2} / \mathrm{m}^{3}$ DO range. This relation indicates that manipulating the $\mathrm{DO}$ value in this range can be an effective way of influencing the cycle duration and the electric power usage. Swarzewo WWTP consists of 4 SBRs. To assess whether, and to what extent, should the performance of one of them be changed, it is needed to manage the entire facility. This work concerns only one reactor, and therefore a constant value of $\mathrm{DO}^{\mathrm{ref}}=2 \mathrm{~g} \mathrm{O}_{2} / \mathrm{m}^{3}$ is assumed. Potential changes of $\mathrm{DO}^{\text {ref }}$ should be managed by the yet higher level controller, responsible for facility optimization.

\section{Control Results}

The aim of the proposed control system was to improve the efficiency of Swarzewo WWTP. 
By this term improvements in overall capacity and effluent quality, as well as cost reduction, are meant. To enable such comparison, reference control algorithm was implemented, in which cycle management was based upon fixed time limits. In order to provide common basis for comparison, the existing PI controller of DO was replaced with the cascade system presented in this paper, so the lower level controller wouldn't affect the results.

The aeration system model and the control algorithm were implemented in MATLAB environment. The SBR was modeled and simulated in SIMBA package.

To ensure that the proposed method is validated within a wide operating range, three cases of pollutant concentrations were considered. Mean values of the parameters of the influent for each case are shown in Table 3. Three cases of the waste inflow rate were also considered: small $3000 \mathrm{~m}^{3} / \mathrm{d}$, medium $-5000 \mathrm{~m}^{3} / \mathrm{d}$, and high $14000 \mathrm{~m}^{3} / \mathrm{d}$. The proposed values were based upon the data from Swarzewo WWTP. The influent temperature was set to $20^{\circ} \mathrm{C}$.

Table 3. Mean values of pollutant concentrations.

\begin{tabular}{|c|c|c|c|}
\hline $\begin{array}{c}\text { Pollutant } \\
\text { concentration }\end{array}$ & $\begin{array}{c}\mathbf{C O D} \\
{\left[\mathbf{g ~ O}_{\mathbf{2}} / \mathbf{m}^{\mathbf{3}}\right]}\end{array}$ & $\begin{array}{c}\mathbf{N}_{\text {tot }} \\
{\left[{\left.\mathbf{g ~ N} / \mathbf{m}^{3}\right]}^{\mathbf{3}}\right.}\end{array}$ & $\begin{array}{c}\mathbf{P}_{\text {tot }} \\
{\left[\mathbf{g ~ P / \mathbf { m } ^ { 3 }}\right]}\end{array}$ \\
\hline case I & 535 & 55 & 7.38 \\
\hline case II & 1070 & 110 & 14.75 \\
\hline case III & 2140 & 220 & 29.5 \\
\hline
\end{tabular}

The results of the simulations are shown in the Table 4. The working volume equal to $V_{\max }$ minus $V_{\min }$, SBR capacity, total energy consumption on aeration, energy consumption per $\mathrm{m}^{3}$ of treated wastewater, COD, $\mathrm{N}_{\text {tot }}$ and $\mathrm{P}_{\text {tot }}$ indicators are included. The tests were conducted for both, reference and proposed supervisory control systems.

The time limits are seasonally determined by the technologist. On the daily basis, the Swarzewo WWTP operator manually adjust aeration time limit on the basis of his observations and experience. This system cannot be implemented in the simulation environment, resulting in the significantly higher nitrogen compounds concentration in the effluent for case III (Table 4), as the time limit was established for medium influent conditions. Even when the manual, on-line control of the aeration phase is applied, it is not always possible to correctly asses needed aeration time, which is reflected by the 12.9 $\mathrm{g} \mathrm{N} / \mathrm{m}^{3}$ annual average $\mathrm{N}_{\text {tot }}$ concentration in the effluent measured in Swarzewo.

In Swarzewo WWTP chemical phosphorus removal with salts of iron is utilized, resulting in annual average $\mathrm{P}_{\text {tot }}$ concentration equal to $0.94 \mathrm{~g} \mathrm{P} / \mathrm{m}^{3}$. The disadvantage of this approach is the increase in the operating costs and increased sludge growth. In this paper possibility of enhanced biological phosphorus removal in Swarzewo is investigated, therefore no iron salts were introduced to reactor. It can be observed that respectively $9.9 \%, 22.2 \%$ and $45.4 \%$ of the phosphorus load was removed in the case of reference work cycle composition and phase management. For the exact same influent conditions, proposed supervisory control algorithm yield much better performance: nearly $96 \%$ of the $\mathrm{N}_{\text {tot }}$ load and $94 \%$ to $86 \%$ of the $\mathrm{P}_{\text {tot }}$ load was removed. Simultaneously, the reactor capacity increased by $88 \%, 68 \%$ and $22 \%$ respectively for three cases, with energy expense decreased by an average of $23 \%$.

Complete reduction of the COD load is the effect of placing aeration phase as the last in the sequence of biological reactions. For this reason the DO concentration in the effluent reaches approximately $0.15 \mathrm{~g} \mathrm{O}_{2} / \mathrm{m}^{3}$ and excess the oxygen demand of the remaining pollutants.

The presented results indicate, that chemical phosphorus removal in Swarzewo is not necessary, enhanced biological phosphorus removal provides similar results. Thereby, the operating costs of the WWTP can be decreased, as the chemical removal of $1 \mathrm{~g} \mathrm{P} / \mathrm{m}^{3}$ can cost anywhere from about $10^{\prime} 000$ to even $35^{\prime} 000 €$ per year, depending on the method of the coagulant preparation.

Further cost reduction is achieved due to decreased electrical energy consumption.

Table 4. The reference / proposed control systems results obtained for medium $\left(5000 \mathrm{~m}^{3} / \mathrm{h}\right)$ inflow rate.

\begin{tabular}{|c|c|c|c|c|c|c|c|}
\hline Case & $\begin{array}{c}\text { Working } \\
\text { volume }\left[\mathbf{m}^{3}\right]\end{array}$ & $\begin{array}{c}\text { Capacity } \\
{\left[\mathbf{m}^{\mathbf{3}} / \mathbf{d}\right]}\end{array}$ & $\begin{array}{c}\text { Energy } \\
{[\mathbf{k W h}]}\end{array}$ & $\begin{array}{c}\text { Energy expense } \\
{\left[\mathbf{k W h} / \mathbf{m}^{3}\right]}\end{array}$ & $\begin{array}{c}\mathbf{C O D} \\
{\left[\mathbf{g} \mathbf{O}_{2} / \mathbf{m}^{3}\right]}\end{array}$ & $\begin{array}{c}\mathbf{N}_{\text {tot }} \\
{\left[\mathbf{g ~ N} / \mathbf{m}^{3}\right]}\end{array}$ & $\begin{array}{c}\mathbf{P}_{\text {tot }} \\
{\left[\mathbf{g ~ P} / \mathbf{m}^{3}\right]}\end{array}$ \\
\hline I & $1793 / 971$ & $1203 / 2266$ & $751 / 317.5$ & $0.4192 / 0.3269$ & $-79 /-81$ & $8 / 2.9$ & $6.6 / 0.48$ \\
\hline II & $1793 / 972$ & $1203 / 2021$ & $1080 / 430.4$ & $0.6023 / 0.4427$ & $-126 /-93$ & $9.2 / 4.3$ & $11.4 / 2.24$ \\
\hline III & $1793 / 970$ & $1203 / 1468$ & $1790 / 755.2$ & $0.9982 / 0.7785$ & $-197 /-117$ & $27.3 / 8.7$ & $16 / 4.24$ \\
\hline
\end{tabular}


Annual energy consumption in Swarzewo in 2011 reached $1.967 \mathrm{MWh}$, with a cost of 0.09 $€ / \mathrm{kWh}$. The total cost of electricity amounted to about $177,000 €, 20 \%$ decrease of electricity consumption would generate up to $35,000 €$ savings per year.

Reference and achieved DO trajectory are shown in the Figure 4. The graph was obtained for a medium flow rate $\left(5000 \mathrm{~m}^{3} / \mathrm{d}\right)$ and case II pollutants concentration. High quality of control for each aeration phase was achieved, despite different pressure and respiratory rate due to step-feed strategy.

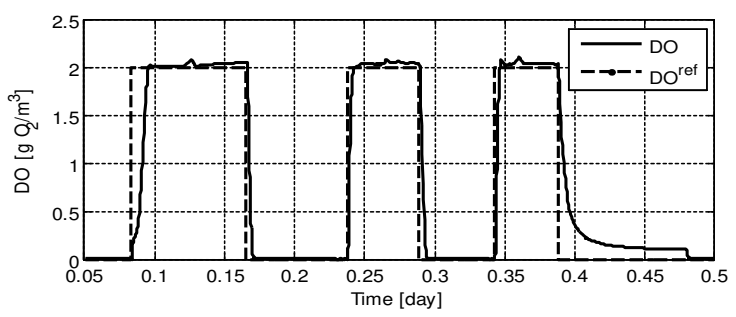

Figure 4. Control results for DO concentration.

The proposed solution was tested over wide range of operating points, including different inflow rate scenarios. The results of simulations are shown in Table 5. Due to work cycle management, the capacity of the SBR is increasing alongside increasing inflow rates. Under normal conditions, 4 working reactors should ensure a capacity of about 8'000-10'000 $\mathrm{m}^{3} /$ day. If the inflow reaches extreme (maximum recorded value) $14,000 \mathrm{~m}^{3} / \mathrm{d}$, the efficiency varies between $6,000-12,000 \mathrm{~m}^{3} / \mathrm{d}$, depending on the amount of pollution. The maximum flow rate is the result of heavy rains, and in such case wastewater is diluted after several minutes, resulting in lower pollutant concentrations. If needed, WWTP capacity can be increased by increasing the DO setpoint.

\section{Conclusions}

The main advantage of the SBR is great operational flexibility, that allows adjustment of the plant to the specific working conditions. However, to fully utilize this potential, the supervisory controller for work cycle and phase management have to be implemented. In the paper design of such controller have been presented. The work cycle composition was discussed with regard to the number of biological reactions sequences and the volume of the last filling. As a result, the strategy of asymmetric filling with variable number of sequences was proposed. Conducted simulations show, that SBR working with that strategy can efficiently deal with highly variable inflow rate, while maintaining high treating performance.

To ensure that oxygen uptake rate during aeration will not exceed aeration system capabilities, the phase management system was designed with technical limitations of the aeration system taken into regard. This concerns, among others: sludge mass, volumetric exchange ratio, filling time or DO reference setpoint.

The optimal duration of biological reaction and sedimentation phases were determined on the basis of nitrogen compounds and TSS measurements, leading to decreased electric power consumption, increased capacity and reduced amount of $\mathrm{P}_{\text {tot }}$ in the effluent. Excellent SBR performance in the terms of $100 \%$ COD reduction, 95\% $\mathrm{N}_{\text {tot }}$ reduction and $85-94 \% \mathrm{P}_{\text {tot }}$ reduction, independently to pollution or inflow rate scenario, was achieved. A the same time SBR operating costs were reduced.

\section{REFERENCES}

1. ÅMAND, L., G. OLSSON, B. CARLSSON, Aeration Control - A Review, Water Sc. Tech., vol. 67(11), 2013, pp. 2374-2398.

2. ARTAN, N., P. WILDERER, D ORHON, et al., Model Evaluation and Optimisation of Nutrient Removal Potential for Sequencing Batch Reactors, Water SA, vol. 28(4), 2002, pp. 423-432.

3. BELCHIOR, C. A. C., R. A. M. ARAÚJO, J.A.C. LANDECK, Dissolved Oxygen Control of the Activated Sludge Wastewater Treatment Process using

Table 5. The results of simulation studies for three cases of pollution and $3 / 5 / 14\left[1000 \mathrm{~m}^{3} / \mathrm{d}\right]$ inflow rates.

\begin{tabular}{|c|c|c|c|c|c|c|}
\hline Case & $\begin{array}{c}\text { Capacity } \\
{\left[\mathbf{m}^{3} / \mathbf{d}\right]}\end{array}$ & $\begin{array}{c}\text { Energy } \\
{[\mathbf{k W h}]}\end{array}$ & $\begin{array}{c}\text { Energy expense } \\
{\left[\mathbf{k W h} / \mathbf{m}^{3}\right]}\end{array}$ & $\begin{array}{c}\mathbf{C O D} \\
{\left[\mathbf{g ~ O}_{2} / \mathbf{m}^{3}\right]}\end{array}$ & $\begin{array}{c}\mathbf{N}_{\text {tot }} \\
{\left[\mathbf{g ~ N}_{\mathbf{m}}\right]}\end{array}$ & $\begin{array}{c}\mathbf{P}_{\text {tot }} \\
{\left[\mathbf{g ~ P / \mathbf { m } ^ { 3 } ]}\right]}\end{array}$ \\
\hline I & $1748 / 2266 / 2965$ & $340 / 318 / 243$ & $0.35 / 0.33 / 0.25$ & $-85 /-81 /-65$ & $2.5 / 2.9 / 2.7$ & $0.5 / 0.5 / 1$ \\
\hline II & $1545 / 2021 / 2244$ & $489 / 430 / 411$ & $0.5 / 0.44 / 0.42$ & $-104 /-93 /-96$ & $4.5 / 4.3 / 5.4$ & $2 / 2.2 / 3.5$ \\
\hline III & $1310 / 1468 / 1505$ & $794 / 755 / 745$ & $0.82 / 0.78 / 0.77$ & $-118 /-117 /-120$ & $8.2 / 8.7 / 10.5$ & $4.8 / 4 / 5.7$ \\
\hline
\end{tabular}


Stable Adaptive Fuzzy Control, Comput. Chem. Eng., vol. 37, 2012, pp. 152-162.

4. BŁASZKIEWICZ, K., R. PIOTROWSKI, K. DUZINKIEWICZ, A Model-Based Improved Control of Dissolved Oxygen Concentration in Sequencing Wastewater Batch Reactor, Stud. Inform. Control, vol. 23 (4), 2014, pp. 323-333.

5. DUZINKIEWICZ, K., R. PIOTROWSKI, M. A. BRDYS, W. KUREK, W., Genetic Hybrid Predictive Controller for Optimized Dissolved-Oxygen Tracking at Lower Control Level, IEEE Trans. Control Syst. Technol., vol. 17(5), 2009, pp. 1183-1192.

6. GARCIA, C. E., M. MORARI, Internal Model Control. A Unifying Review and Some New Results, Ind. Eng. Chem. Proc. Des. Dev., vol. 21(2), 1982, pp. 308-323.

7. HAN, H.-G., J.-F. QIAO, Q.-L., CHEN, Model Predictive Control of Dissolved Oxygen Concentration based on a Selforganizing RBF Neural Network, Control Eng. Pract., vol. 20(4), 2012, pp. 465-476.

8. HENZE, M., W. GUJER, T. MINO, M. van LOOSDRECHT, Activated Sludge Models ASM1, ASM2, ASM2d and ASM3. London: IWA Publ, 2007.

9. HIRSCH, P., R. PIOTROWSKI, K. DUZINKIEWICZ, Two-step Model based Adaptive Controller for Dissolved Oxygen Control in Sequencing Wastewater Batch Reactor. MMAR 2015. IEEE, 2015, pp. 677-682.

10. PAVŠELJ, N., N. HVALA, J. KOCIJAN, et al., Experimental Design of an Optimal Phase Duration Control Strategy used in Batch Biological Wastewater Treatment, ISA Trans., vol. 40(1), 2001, pp. 41-56.

11. PIOTROWSKI, R., M. A. BRDYS, K. KONARCZAK, et al., Hierarchical Dissolved Oxygen Control for Activated Sludge Processes, Control Eng. Pract., vol. 16(1), 2008, pp. 114-131.

12. PIOTROWSKI, R., Two-Level Multivariable Control System of Dissolved Oxygen Tracking and Aeration
System for Activated Sludge Processes, Water Env. Res., vol. 87(1), 2015, pp. 3-13.

13. PIOTROWSKI, R., K. BŁASZKIEWICZ, K. DUZINKIEWICZ, Analysis the Parameters of the Adaptive Controller for Quality Control of Dissolved Oxygen Concentration, Inf. Tech. Control, vol. 45(1), 2016, pp. 42-51.

14. PIOTROWSKI, R., A. SKIBA, Nonlinear Fuzzy Control System for Dissolved Oxygen with Aeration System in Sequencing Batch Reactor, Inf. Tech. Control, vol. 44(2), 2015, pp. 182-195.

15. SANTÍN, I., C. PEDRET, R. VILANOVA, Applying Variable Dissolved Oxygen Set Point in a Two Level Hierarchical Control Structure to a Wastewater Treatment Process, J. Proc. Ctrl, vol. 28, 2015, pp. 40-55.

16. SIN, G., G. INSEL, D. S. LEE, P.A. VANROLLEGHEM, Optimal But Robust $N$ and $P$ Removal in SBRs: $A$ Modelbased Systematic Study of Operation Scenarios, Water Sci. Tech., vol. 50(10), 2004, pp. 97-105.

17. TRAORÉ, A., L. COROMINAS, S. PUIG, et al., Dissolved Oxygen Control and Phases Duration Optimization in a Sequencing Batch Reactor Pilot Plant. Proc. 17th IMACS World Congress on Scientific Computation. France, Applied Mathematics and Simulation, 2005.

18. VREČKO, D., N. HVALA, M. STRAŽAR, The Application of Model Predictive Control of Ammonia Nitrogen in an Activated Sludge Process, Water Sci. Tech., vol. 64(5), 2011, pp. 1115.

19. WAHAB, N. A., R. KATEBI, J. BALDERUD, Multivariable PID Control Design for Activated Sludge Process with Nitrification and Denitrification, Bioch. Eng. J., vol. 45(3), 2009, pp. 239-248.

20. YANG, T., W. QIU, Y. MA, et al., Fuzzy Model-based Predictive Control of Dissolved Oxygen in Activated Sludge Processes, Neurocomputing, vol. 136, 2014, pp. 88-95. 